\title{
Interactive comment on "Tree-ring based spring precipitation reconstruction in the Sikhote-Alin Mountain Range" by Olga Ukhvatkina et al.
}

\section{Olga Ukhvatkina et al.}

ukhvatkina@gmail.com

Received and published: 30 September 2020

Dear Reviewer, We would like to thank you for your careful analysis of our manuscript and your valuable suggestions that have helped improve it. Please, find below our answers to your comments.

1) The authors state that they sampled trees in an area where almost no anthropogenic activity occurred over the last 300 to 500 years, this is really interesting. Do the authors think that it could be possible to extend back in time the existing records? I would discuss somewhere in the discussion whether it would be possible to extend the chronology back in time using living, dead and/or subfossil materials. I think that most of the reader never had the chance to go the Sikhote-Alin Mountain Range. Would it 
be possible to add to figure 1 a picture of the study site and possibly a picture of one disc collected by the authors?

In this study area, we can only use cores from living trees and discs (usually fragments of them) of a few dead Pinus koraiensis trees for several reasons. First, due to high humidity in summer (in the forest the air humidity during the summer is close to 100\%) wood decomposes very quickly, so it is very difficult to find a well-preserved dead tree. Secondly, the old wooden buildings are completely absent. Finally, sub-fossil trees are extremely rare and are found only in one location within the study area - not far the NSA. Therefore, taking into account the maximum age of Pinus koraiensis trees and the rate of wood decomposition, we believe that the maximum length of chronologies can be about 600-700 years. We are currently collecting additional data in order to increase the length of the chronologies (especially for the NSA, where the chronology is relatively short). We've added this information to the Discussion. We think it's a good idea to add some photos, we added two to Figure 1, and will add more in the Supplement.

2) Could all the samples collected be crossdated?

Yes, this is possible and makes sense if we want to obtain a regional chronology. In our case, we decided that it would be better if we make separate chronologies for each site. First, the reference years important for crossdating at different points often do not coincide. Second, tree ring data from trees in a closed canopy forest is usually "noisy" due to relationships between trees. Therefore, crossdating such data from remote locations is a rather difficult task. In general, the result of crossdating all data will be less accurate than crossdating data from individual sample sites (we tried it).

3) This concern has already been raised by other referees, but it would really useful to have more details about the detrending method used by the authors. Age-dependent spline smoothing is a very general description. The author should keep in mind that Science should always be reproducible and in this respect providing sufficient details

Interactive comment 
for the reader to understand how the analyses were performed is really important. Could the authors let us know the reasons that led them choose this particular method over other methods such the negative exponential method for instance?

To be more precise, in ARSTAN we used a 60-years low-pass filter for smoothing. We added this information to our manuscript. As for the choice of a specific detrending method. When a tree grows alone (without interaction with other trees, for example, at the top of a high mountain), then its growth, both in height and in diameter, is well described by an S-shaped curve: at first tree growth is relatively slow, then it accelerates, and finally it slows down again. In this case, a negative exponential curve is good choice for detrending. However, if a tree grows in a closed canopy stand (like all trees in our study), then it usually has several abrupt growth increases (so-called "releases"), after which growth slows down. Therefore, in this case, the cubic smoothing line is better suited. We have added a short explanation to the manuscript.

4) How did you aggregate the detrended series together? Did you use the Tukey's Robust Mean or simply averaged the detrended series together?

This is also done in ARSTAN. By default, ARSTAN uses robust mean and we use it. One can also choose the arithmetic mean, but we don't think anyone is changing this option (since using robust mean is integral part of ARSTAN). To be more precise, we have added information about robust mean to the manuscript.

5) Did the authors account for variance changes resulting from changing sampled replication?

As one of the descriptions of the ARSTAN says, the index values (obtained as a result of standardization) are unitless, with a nearly stable mean and variance, allowing indices from numerous trees to be averaged into a site chronology. We think this is also true for changing sampled replication; ARSTAN has no additional settings for this.

6) Overall I think that the section "Tree-Ring Chronology development" could be ex-

Interactive

comment
Printer-friendly version

Discussion paper 
panded slightly and should contain more details.

We have expanded this section in accordance with your comments.

7) Lines 132-1322, the author state: "A correlation analysis was used to evaluate the relationships between the ring-width index and observed monthly climate records from the previous June to the current September" Did the authors used bootstrapped correlations functions? Again, additional details would be most welcome.

Indeed, treeclim uses bootstrapping to test for significant correlations and there are several different options for that. We've added clarifying information to the manuscript.

8) I concur with the other referee that, using the residual chronology to perform climate reconstruction is a little bit unusual... Have the author at least tried to perform the precipitation reconstructions using the standard chronologies? Do the reconstructions have some predictive skills? One compromise could be to present the "residual reconstructions" in the main manuscript and to present the "standard reconstructions" in the supplementary material.

Of course, we tried to reconstruct precipitation using both residual and standard chronologies. In general, standard chronologies had a lower sensitivity and correlated worse with precipitation (after comments from previous reviewers, we added to Supplement a figure similar to Figure 4, but for the standard chronologies). Predictive skills of the residual chronologies also were better. In addition, for standard chronology CSA we got $\mathrm{CE}<0$ and so we cannot use it for precipitation reconstruction in this particular case. We think it is a good idea to add "standard reconstructions" to the Supplement. We've added standard chronologies for three sample sites and two reconstructions for SSA and NSA.

9) Unless I missed something, I was not able to locate the error bars in the plots displaying the reconstructions. The authors should keep in mind that trees are not perfect rain gauges. The method used to reconstruct precipitation variability also comes with lim-

Interactive comment
Printer-friendly version

Discussion paper 
itations. Therefore paleoclimatic reconstruction should always come with uncertainty estimates. I would also invite the author to describe in the method section how they computed the uncertainties of the reconstructions.

We have added uncertainty bans to Figures 5 and 6; estimated as twice the standard error of prediction $( \pm 2 \sigma)$ (Wilks, 1995)

10) Figure 3: I would not reconstruct precipitation for the sections of the chronologies having an EPS below 0.85 .

\section{Corrected.}

11) Figure 3, 5, and 6: Whenever possible I would encourage the authors to use the exact same scale for the $Y$ axis.

In Figure 3, we changed the " $Y$ " axes so that they became same for all graphs (all three sample sites). In Figures 5 and 6, we made the same " $Y$ " axes for SSA and CSA, but did not change the axis for NSA, since for this site we reconstructed precipitation for a much longer season (March-July) than for the other two points. Accordingly, if we make the same "Y" scale on the graphs with reconstructions, then the reconstructions for SSA and CSA will look flat.

12) There is something odd in the Table 2. RE and CE are replaced by $E$ and $E$.

Corrected (also something odd happened with DW)

13) I do also have a few concerns about the authors' conclusions regarding the linkages with ENSO and PDO... The author didn't find any significant relationships with the NINO3, NINO4, NINO3.4 and SOI indexes, yet they hypothesize that the periodicities detected by the wavele analyses are related to ENSO. . . How can the authors be sure that the 3 years cycle is related to ENSO? It could be something completely different. I am not sure that the evidence currently presented by the authors support their conclusions. Providing more details regarding the influence of ENSO on Far East Russia would be also be welcome. If I am not mistaken, so far the authors only cited 
one reference (Byshev et al., 2014). Does it mean that no other study attempted to investigate the influence of ENSO on Far East Russia's climate?

Indeed, we found no significant correlations between our reconstructions and the ENSO indices. Of course, having received such results, we began to think about what caused them (since, as we assumed, there should be some relationships between precipitation and ENSO and PDO). We analyzed the relationship between the ENSO and PDO indices by instrumental records and found that if we consider the entire period of the summer monsoon, then significant correlations can be found, but if we consider only the first part of the monsoon, then there are no significant correlations. Therefore, the influence of ENSO and PDO (also AO) actually exists, but we do not detect it in our reconstructions, since we are reconstructing precipitation for the first part of the summer monsoon. The only possible evidence of this effect that we have obtained is the cycles identified using wavelet analysis. We agree with you, even though these cycles are similar to the influence of ENSO and PDO, it could be something completely different. Therefore, we changed the phrases about the effect of oscillations in the conclusion so that it sounds not like a proven fact, but like our assumption. We also cite Ponomarev et al, 2015 (Features of climate regimes in the North Asian Pacific). Of course, we were looking for works where the influence of ENSO and other oscillations on the studied region would be investigated, but practically nothing was found. There are separate studies for other parts of the Russian Far East, but this is a huge territory (the distance between the southern and northern points is about $4000 \mathrm{~km}$ ) and the climate in different parts is completely different. Therefore, yes, we cannot cite other works, at least we could not find them.

Please also note the supplement to this comment: https://cp.copernicus.org/preprints/cp-2020-49/cp-2020-49-AC3-supplement.pdf

Interactive comment on Clim. Past Discuss., https://doi.org/10.5194/cp-2020-49, 2020. 